\title{
Optical inverse-Compton emission from clusters of galaxies
}

\section{Citation}

Yamazaki, Ryo, and Abraham Loeb. 2015. "Optical Inverse-Compton Emission from Clusters of Galaxies." Monthly Notices of the Royal Astronomical Society 453 (2): 1990-98. https:// doi.org/10.1093/mnras/stv1757.

\section{Permanent link}

http://nrs.harvard.edu/urn-3:HUL.InstRepos:41412161

\section{Terms of Use}

This article was downloaded from Harvard University's DASH repository, and is made available under the terms and conditions applicable to Open Access Policy Articles, as set forth at http:// nrs.harvard.edu/urn-3:HUL.InstRepos:dash.current.terms-of-use\#OAP

\section{Share Your Story}

The Harvard community has made this article openly available.

Please share how this access benefits you. Submit a story.

Accessibility 
Mon. Not. R. Astron. Soc. 000,111(2015) Printed 9 October $2018 \quad$ (MN LATEX style file v2.2)

\title{
Optical inverse-Compton emission from clusters of galaxies
}

\author{
Ryo Yamazaki ${ }^{1,2 \star}$ and Abraham Loeb ${ }^{2}$ \\ ${ }^{1}$ Department of Physics and Mathematics, Aoyama Gakuin University, 5-10-1, Fuchinobe, Sagamihara 252-5258, Japan \\ ${ }^{2}$ Harvard-Smithsonian Center for Astrophysics, 60 Garden Street, Cambridge, MA, 02138, USA
}

9 October 2018

\begin{abstract}
Shocks around clusters of galaxies accelerate electrons which upscatter the Cosmic Microwave Background photons to higher-energies. We use an analytical model to calculate this inverse Compton (IC) emission, taking into account the effects of additional energy losses via synchrotron and Coulomb scattering. We find that the surface brightness of the optical IC emission increases with redshift and halo mass. The IC emission surface brightness, 32-34 mag $\operatorname{arcsec}^{-2}$, for massive clusters is potentially detectable by the newly developed Dragonfly Telephoto Array.
\end{abstract}

Key words: acceleration of particles — galaxies: clusters: general — intergalactic medium — radiation mechanisms: nonthermal

\section{INTRODUCTION}

According to the standard model of hierarchical structure formation, accretion shocks occur around the virial radii of massive clusters (e.g., Ryu et al. 2003; Schaal \& Springel 2015, and references therein). In these shocks, electrons are expected to be accelerated by first-order Fermi mechanism (see, e.g., Brunetti \& Jones 2014, for recent review) to have power-law distribution (Blandford \& Ostriker 1978; Bell 1978). Turbulence in the intergalactic medium (IGM; Rvu et al. 2008; Takizawa 2008; Miniati 2015) is another source of particle acceleration via second-order Fermi mechanism (Schlickeiser et al. 1987; Brunetti et al. 2001; Petrosian 2001; Fuiita et al. 2003). Relativistic electrons give rise to inverse Compton (IC) emission by upscattering Cosmic Microwave Background (CMB) photons as well as radio synchrotron emission. So far, IC emission has been studied mainly in hard X-ray and gammaray bands both theoretically and observationally (Sarazin 1999; Loeb \& Waxman 2000; Totani \& Kitayama 2000; Takizawa \& Naito 2000; Fuiita \& Sarazin 2001; Takizawa 2002; Keshet et al. 2003, 2004a, 2012; Petrosian et al. 2008; Kushnir \& Waxman 2010; Bartels et al. 2015). However, currently there are only upper limits (Ota et al. 2014; Gastaldello et al. 2015, and references therein) and a few claimed detections of hard X-rays (Rephaeli et al. 2008), but no detection in the gamma-ray band (Ackermann et al. 2010, 2014), which may constrain particle acceleration mechanisms (e.g., Zandanel \& Ando 2014; Vazza et al. 2015). Although IC emission from individual clusters has not yet

* E-mail: ryo@phys.aoyama.ac.jp been detected, the cumulative emission from all of them may contribute to extragalactic gamma-ray background Loeb \& Waxman 2000; Miniati 2002).

In this paper, we focus on the IC emission namely in the optical band. So far, it has been thought that the optical IC emission is too dim to be detectable (e.g., Sarazin 1999; Fujita \& Sarazin 2001). However, an advanced technique has recently been developed in the form of the Dragonfly Telephoto Array Abraham \& van Dokkum 2014; van Dokkum et al. 2014), which is optimized for the detection of extended ultra low surface brightness structures and is capable of imaging extended structures to surface brightness levels below $32 \mathrm{mag} \operatorname{arcsec}^{-2}$ in the SDSS g band with a reasonable exposure time. This newly developed technique provides a new motivation for calculating the brightness of the optical IC emission in detail. Since no firm detection of IC emission at any wavelength has been reported as of yet, the optical telescopes hold the potential to bring the first clear detection of the IC emission from large-scale shocks around clusters of galaxies, which is also the first evidence of nonthermal processes at accretion shocks.

We construct a simple one zone, analytical model for IC emission from cluster shocks, allowing us to capture the essential physical details as well as the parameter dependence of the results. Because the electrons emitting the optical IC emission have the Lorentz factor $\sim 50$, they are potentially affected by Coulomb energy losses (e.g., Sarazin 1999; Petrosian et al. 2008). However, as we show later, this effect is not significant. Our model applies also to other sources of nonthermal electrons. We focus on IC from primarily accelerated electrons for simplicity, although secondary electrons may also contribute (e.g., Blasi \& Colafrancesco 1999; 
Miniati 2003; Inoue et al. 2005; Kushnir \& Waxman 2009). For the small magnetic field strength expected in clusters, the contribution of synchrotron emission to the optical brightness is negligible.

Our paper is organized as follows. In section 2, we describe our analytical model for calculating the IC emission. The surface brightness in the SDSS g-band is then calculated for fiducial parameters in section 3. Finally, we summarize our results and predictions for specific clusters in section 4 . Throughout the paper, we assume a flat $\Lambda$ CDM universe with cosmological parameters, $h=0.6774, \Omega_{\mathrm{m}}=0.3089$, $\Omega_{\Lambda}=0.6911$, and $\Omega_{\mathrm{b}}=0.0486$ (Ade et al. 2015)

\section{ANALYTICAL MODEL OF IC SPECTRUM}

\subsection{Physical quantities of cluster shocks}

A halo of mass $M$ collapsing at redshift $z$ has a virial radius $r_{\text {vir }}$, within which the mean density is $\Delta_{\mathrm{c}}$ times the critical density $\rho_{\mathrm{c}}(z)$, and circular velocity at the virial radius $V_{\mathrm{c}}$ given by (Brvan \& Norman 1998; Barkana \& Loeb 2001),

$$
\begin{aligned}
r_{\mathrm{vir}} & =\left(\frac{3 M}{4 \pi \Delta_{\mathrm{c}} \rho_{\mathrm{c}}(z)}\right)^{1 / 3} \\
& =0.79 h^{-2 / 3} M_{14}^{1 / 3} w(z)^{-1 / 3}(1+z)^{-1} \mathrm{Mpc} \\
V_{\mathrm{c}} & =\sqrt{\frac{G M}{r_{\mathrm{vir}}}} \\
& =7.4 \times 10^{7} h^{1 / 3} M_{14}^{1 / 3} w(z)^{1 / 6}(1+z)^{1 / 2} \mathrm{~cm} \mathrm{~s}^{-1}
\end{aligned}
$$

where $M_{14}=\left(M / 10^{14} M_{\odot}\right)$. The function $w(z)$ is given by

$w(z)=\frac{\Omega_{\mathrm{m}}}{\Omega_{\mathrm{m}}^{z}} \frac{\Delta_{\mathrm{c}}}{18 \pi^{2}}$,

where

$\Omega_{\mathrm{m}}^{z}=\frac{\Omega_{\mathrm{m}}(1+z)^{3}}{\Omega_{\mathrm{m}}(1+z)^{3}+\Omega_{\Lambda}}$,

$\Delta_{\mathrm{c}}=18 \pi^{2}+82\left(\Omega_{\mathrm{m}}^{z}-1\right)-39\left(\Omega_{\mathrm{m}}^{z}-1\right)^{2}$.

Note that $w(z)$ is a monotonically decreasing function of $z$, starting at $w(0)=0.58$, decaying through $w(1)=0.35$ and asymptotically approaching $w(z) \rightarrow \Omega_{\mathrm{m}}=0.31$ as $z \rightarrow \infty$.

For simplicity, we assume that a spherical virial shock is formed at $r_{\mathrm{vir}}$, and that accretion is smooth and not associated with mergers of sub-units. Recent numerical simulations have shown that accretion shocks are deformed and far from spherical (e.g., Rvu et al. 2003; Lau et al. 2015; Schaal \& Springel 2015; Nelson et al. 2015), however, emsemble-averaged gas profile shows that virial shocks exist near the virial radius. Future extensions of this work can be based on numerical simulations of non-spherical configurations. The ensemble-averaged mass accretion rate onto the halo is written as (White 1994),

$\dot{M}=f_{\text {acc }} \frac{V_{\mathrm{c}}^{3}}{G}$,

and the shock temperature is given by,

$$
k T=f_{\mathrm{T}} \mu m_{p} V_{\mathrm{c}}^{2}
$$

where $\mu m_{p}$ is the average mass of a particle (including electrons), and we adopt $\mu=0.6$. The factors $f_{\text {acc }}$ and $f_{\mathrm{T}}$ are dimensionless numbers of order unity. The gas density just in front of the shock can be written as,

$$
\begin{aligned}
n_{g} & =\frac{\left(\Omega_{\mathrm{b}} / \Omega_{\mathrm{m}}\right) \dot{M}}{4 \pi r_{\mathrm{vir}}^{2} V_{\mathrm{c}} \mu m_{p}} \\
& =8.0 \times 10^{-5} f_{\mathrm{acc}} w(z)(1+z)^{3} \mathrm{~cm}^{-3} .
\end{aligned}
$$

This estimate is roughly consistent with the results given by Patej \& Loeb (2015) if $f_{\text {acc }} \approx 0.5$. The gas is compressed at the shock with shock compression ratio $r$, which is somewhat uncertain. Recent numerical simulations have shown that the shocks are not so strong and their typical Mach number is around a few (Rvu et al. 2003; Schaal \& Springel 2015), i.e., $r<4$. The IGM is pre-heated and the shocks are not so strong at present epoch $z \sim 0$, however, at high redshifts $z \gtrsim 1$ when the IGM is cold, the shocks around clusters are likely to be strong $(r \approx 4)$. Fortunately, we will see in section 3 that the IC flux does not depend on $r$ for fiducial parameters.

The magnetic field around the shock is amplified as in supernova remnants (e.g., Vink \& Laming 2003; Bamba et al. 2003, 2005a b). Assuming that the energy density of the downstream magnetic field constitutes a fraction $\xi_{\mathrm{B}}$ of the downstream thermal energy density, we estimate the magnetic field strength as (Waxman \& Loeb 2000; Keshet et al. 2004b; Fuiita \& Kato 2005; Kushnir \& Waxman 2009),

$$
\begin{aligned}
B & =\left(12 \pi r \xi_{\mathrm{B}} n_{g} f_{\mathrm{T}} \mu m_{p}\right)^{1 / 2} V_{\mathrm{c}} \\
& =0.72\left(r_{4} f_{\mathrm{T}} f_{\mathrm{acc}} \xi_{\mathrm{B},-2}\right)^{1 / 2} M_{14}^{1 / 3} w(z)^{2 / 3}(1+z)^{2} \mu \mathrm{G}(9)
\end{aligned}
$$

where $\xi_{\mathrm{B},-2}=\left(\xi_{\mathrm{B}} / 0.01\right)$ and $r_{4}=(r / 4)$.

\subsection{Injected electron spectrum}

We assume a single power-law form of injected electrons

$\dot{N}(\gamma)=N_{0} \gamma^{-p}, \quad\left(\gamma_{\min }<\gamma<\gamma_{\max }\right)$,

with a constant normalization $N_{0}$ and a spectral index $p$.

The maximum Lorentz factor, $\gamma_{\max }$, is determined by the balance of acceleration time and the cooling time. The acceleration time is given by (Drurv 1983),

$t_{\mathrm{acc}}(\gamma)=\frac{r(r+1)}{r-1} \frac{\eta_{g} \gamma m_{e} c^{3}}{e B v_{\mathrm{sh}}^{2}}$,

where the gyro-factor $\eta_{g}$ is of order unity, and the shock velocity that is measured in the rest frame of the shock, $v_{\mathrm{sh}}$, is related to $V_{\mathrm{c}}$ through $v_{\mathrm{sh}}=[r /(r-1)] V_{\mathrm{c}}$. Here we assume Bohm diffusion with no change in the diffusion properties across the shock, and no shock modification due to accelerated particles. We equate $t_{\text {acc }}$ to the cooling time via synchrotron and IC emission,

$t_{\mathrm{IC} / \mathrm{syn}}(\gamma)=\frac{6 \pi m_{e} c}{\sigma_{\mathrm{T}}\left(B^{2}+B_{\mathrm{CMB}}^{2}\right) \gamma}$,

where $B_{\mathrm{CMB}}=3.24(1+z)^{2} \mu \mathrm{G}$, to obtain Loeb \& Waxman 2000),

$$
\begin{aligned}
\gamma_{\max }= & 3.4 \times 10^{7} r_{4}^{3 / 4}\left(\frac{15}{r^{2}-1}\right)^{1 / 2} \\
& \times \frac{\left(f_{\mathrm{T}} f_{\mathrm{acc}} \xi_{\mathrm{B},-2}\right)^{1 / 4} M_{14}^{1 / 2}}{\eta_{g}^{1 / 2}\left[1+\left(B / B_{\mathrm{CMB}}\right)^{2}\right]^{1 / 2}} \frac{w(z)^{1 / 2}}{(1+z)^{1 / 2}}
\end{aligned}
$$


based on Eqs. (2) and (9). We note that the effect of synchrotron cooling is negligible since,

$$
\left(\frac{B}{B_{\mathrm{CMB}}}\right)^{2}=4.9 \times 10^{-2} r_{4} f_{\mathrm{T}} f_{\mathrm{acc}} \xi_{\mathrm{B},-2} M_{14}^{2 / 3} w(z)^{4 / 3},
$$

is always small for our adopted parameters.

In order to determine both the normalization constant $N_{0}$ and the minimum Lorentz factor $\gamma_{\min }$, we make two assumptions. One is that the production rate of the accelerated electrons is a fraction $\eta_{e}$ of the particle number input rate across the virial shock, $\dot{N}_{\text {in }}=\left(\Omega_{\mathrm{b}} / \Omega_{\mathrm{m}}\right) \dot{M} / \mu m_{p}$. The other is that a fraction $\xi_{e}$ of thermal shock energy $(3 / 2) k T \dot{N}_{\text {in }}$ is carried by relativistic electrons. These conditions can be written as

$$
\begin{aligned}
& \int_{\gamma_{\min }}^{\gamma_{\max }} \dot{N}(\gamma) d \gamma=\eta_{e} \dot{N}_{\mathrm{in}} \\
& \int_{\gamma_{\min }}^{\gamma_{\max }} \gamma m_{e} c^{2} \dot{N}(\gamma) d \gamma=\xi_{e} \frac{3}{2} k T \dot{N}_{\mathrm{in}} .
\end{aligned}
$$

One can solve these two equations numerically for $N_{0}$ and $\gamma_{\min }$, given $p, \gamma_{\max }, \eta_{e}$ and $\xi_{e}$. For our fiducial parameter set, $\gamma_{\min }$ is much smaller than $\gamma_{\max }$ and if $p>2$ it is approximately given by,

$$
\begin{aligned}
\gamma_{\min } & \approx \frac{3(p-2) k T}{2(p-1) \eta_{e} m_{e} c^{2}} \\
& =8.4 \frac{(p-2) \xi_{e,-2}}{(p-1) \eta_{e,-5}} f_{\mathrm{T}} M_{14}^{2 / 3} w(z)^{1 / 3}(1+z),
\end{aligned}
$$

where $\xi_{e,-2}=\left(\xi_{e} / 0.01\right)$ and $\eta_{e,-5}=\left(\eta_{e} / 10^{-5}\right)$. For our fiducial parameters, this approximate formula is accurate enough as long as $p>2.2$. Finally, for convenience, Eq. (16) can be written as,

$N_{0} m_{e} c^{2}=\frac{\xi_{e}}{f(p)} \frac{3}{2} k T \frac{\left(\Omega_{\mathrm{b}} / \Omega_{\mathrm{m}}\right) \dot{M}}{\mu m_{p}}$,

where

$f(p)=\left\{\begin{array}{ll}\ln \left(\gamma_{\max } / \gamma_{\min }\right) & (p=2) \\ \frac{1}{p-2} \gamma_{\min }^{2-p}\left[1-\left(\gamma_{\min } / \gamma_{\max }\right)^{p-2}\right] & (p \neq 2)\end{array}\right.$.

\subsection{Spectrum of IC emission}

Next we derive analytically the radiation spectrum of the IC emission for a power-law distribution of injected electrons given by Eq. (10). A similar analysis has been done for synchrotron radiation in the study of gamma-ray bursts (e.g., Sari, Piran, \& Naravan 1998).

The radiation power and the characteristic frequency of upscattered CMB photons which is radiated from a relativistic electron with Lorentz factor $\gamma$ are (Blumenthal \& Gould 1970),

$$
\begin{aligned}
P(\gamma) & =\frac{4}{3} \sigma_{\mathrm{T}} c \gamma^{2} \frac{B_{\mathrm{CMB}}^{2}}{8 \pi} \\
\nu(\gamma) & =\frac{4}{3} \gamma^{2} \bar{\nu}_{\mathrm{CMB}} \\
& =2.05 \times 10^{11} \gamma^{2}(1+z) \mathrm{Hz}
\end{aligned}
$$

where $h \bar{\nu}_{\mathrm{CMB}} \approx 2.70 k T_{\mathrm{CMB}}$ is the mean energy of $\mathrm{CMB}$ photons and we adopt $T_{\mathrm{CMB}}=2.726(1+z) \mathrm{K}$ (Fixsen 2009). The spectral power, $P_{\nu}$ (power per unit frequency, in units of ergs $\left.\mathrm{s}^{-1} \mathrm{~Hz}^{-1}\right)$, is proportional to $\nu$ for $\nu<\nu(\gamma)$ and cuts off sharply at $\nu>\nu(\gamma)$. The function $P_{\nu}$ is peaked around $\nu(\gamma)$, and its peak value is well approximated as $P_{\nu, \max } \approx P(\gamma) / \nu(\gamma)$. Note that $P_{\nu, \max }$ is independent of $\gamma$.

The above description of $P_{\nu}$ is only suitable when the electron does not lose a significant fraction of its energy to radiation. This requires the characteristic cooling time of the electrons to be longer than the dynamical time of a cluster, which is given by,

$t_{\mathrm{dyn}}=\frac{r_{\mathrm{vir}}}{V_{\mathrm{c}}}=1.5 w(z)^{-1 / 2}(1+z)^{-3 / 2} \mathrm{Gyr}$.

Otherwise, the effect of energy loss must be considered. The energy loss rate of electrons is dominated by Coulomb collisions at low energies and synchrotron and IC losses at high energies (Sarazin 1999; Petrosian et al. 2008). The cooling time via Coulomb collisions is well approximated for relativistic electrons as,

$t_{\mathrm{Clmb}}(\gamma)=\frac{2}{3 \sigma_{\mathrm{T}} c n_{e} \ln \Lambda} \gamma$

In the following, we assume that the electron density downstream of the shock is $n_{e} \approx 0.5 r n_{g}$, where the factor 0.5 represents the number fraction of electrons to total gas particles, and the gas density $n_{g}$ is given in Eq. (8). For simplicity, we fix a Coulomb logarithm at a value $\ln \Lambda=40$. The synchrotron and IC cooling times have been already derived in Eq. (12). One can find the Lorentz factors, $\gamma_{\mathrm{b} 1}$, $\gamma_{\mathrm{b} 2}$ and $\gamma_{\mathrm{b} 3}$, at which two of the three timescales, $t_{\mathrm{IC} / \mathrm{syn}}(\gamma)$, $t_{\mathrm{IC} / \mathrm{syn}}(\gamma)$ and $t_{\mathrm{dyn}}$, are balanced, such that,

$$
\begin{aligned}
t_{\mathrm{IC} / \mathrm{syn}}\left(\gamma_{\mathrm{b} 1}\right) & =t_{\mathrm{dyn}} \\
t_{\mathrm{Clmb}}\left(\gamma_{\mathrm{b} 2}\right) & =t_{\mathrm{IC} / \mathrm{syn}}\left(\gamma_{\mathrm{b} 2}\right) \\
t_{\mathrm{Clmb}}\left(\gamma_{\mathrm{b} 3}\right) & =t_{\mathrm{dyn}}
\end{aligned}
$$

Since $t_{\mathrm{IC} / \mathrm{syn}} \propto \gamma^{-1}, t_{\mathrm{Clmb}} \propto \gamma$ and $t_{\mathrm{dyn}} \propto \gamma^{0}, \gamma_{\mathrm{b} 2}$ is always between $\gamma_{\mathrm{b} 1}$ and $\gamma_{\mathrm{b} 3}$. Electrons with Lorentz factor $\gamma$ do not suffer significant cooling only if $\gamma_{\mathrm{b} 3}<\gamma<\gamma_{\mathrm{b} 1}$.

To find the spectral shape of the IC emission taking into account the electron cooling, we define characteristic frequencies as,

$\nu_{\mathrm{b} i}=\nu\left(\gamma_{\mathrm{b} i}\right) \quad, \quad i=1,2,3$

and obtain,

$$
\begin{aligned}
\nu_{\mathrm{b} 1} & =4.8 \times 10^{17} \frac{w(z)(1+z)^{-4}}{\left[1+\left(B / B_{\mathrm{CMB}}\right)^{2}\right]^{2}} \mathrm{~Hz}, \\
\nu_{\mathrm{b} 2} & =2.9 \times 10^{15}\left(\frac{\ln \Lambda}{40}\right) \frac{r_{4} f_{\mathrm{acc}} w(z)}{1+\left(B / B_{\mathrm{CMB}}\right)^{2}} \mathrm{~Hz}, \\
\nu_{\mathrm{b} 3} & =1.8 \times 10^{13}\left(\frac{\ln \Lambda}{40}\right)^{2} r_{4}^{2} f_{\mathrm{acc}}^{2} w(z)(1+z)^{4} \mathrm{~Hz} .
\end{aligned}
$$

These three frequencies coincide at a redshift $z_{\text {eq }}$ approximately given by,

$1+z_{\mathrm{eq}} \approx 3.6\left(\frac{\ln \Lambda}{40}\right)^{-1 / 4}\left(r_{4} f_{\mathrm{acc}}\right)^{-1 / 4}$

where the term $\left(B / B_{\mathrm{CMB}}\right)^{2}$ is small and hence neglected [see Eq. (14)]. One can see $\nu_{\mathrm{b} 3}<\nu_{\mathrm{b} 2}<\nu_{\mathrm{b} 1}$ for $z<z_{\text {eq }}$ and $\nu_{\mathrm{b} 1}<\nu_{\mathrm{b} 2}<\nu_{\mathrm{b} 3}$ for $z>z_{\mathrm{eq}}$. The spectral shapes are different for these two cases, and are treated separately in the following. Our results are summarized in Figure 1 . 


\subsubsection{IC spectrum for $\nu_{\mathrm{b} 3}<\nu_{\mathrm{b} 2}<\nu_{\mathrm{b} 1}$}

An electron with an initial Lorentz factor $\gamma>\gamma_{\mathrm{b} 1}$ cools down to $\gamma_{\mathrm{b} 1}$ in the dynamical time $t_{\mathrm{dyn}}$. Recall that the peak value of the instantaneous emissivity, $P_{\nu, \max }$, is independent of the electron energy. Thus, the average emission power at frequency $\nu$ is proportional to the cooling time of electrons with Lorentz factor $\gamma$, satisfying $\nu=\nu(\gamma) \propto \gamma^{2}$. Therefore, the average spectrum scales a\& $P_{\nu} \propto t_{\mathrm{IC} / \mathrm{syn}}(\gamma) \propto \nu^{-1 / 2}$ for $\nu_{\mathrm{b} 1}<\nu<\nu(\gamma)$. For $\nu<\nu_{\mathrm{b} 1}$, the spectrum has a low-energy tail, $P_{\nu} \propto \nu$. For $\nu>\nu(\gamma)$, the spectrum steeply decays. The averaged spectrum from such electrons has a peak at $\nu_{\mathrm{b} 1}$.

An electron with an initial Lorentz factor $\gamma_{\mathrm{b} 3}<\gamma<\gamma_{\mathrm{b} 1}$ does not suffer significant cooling, and the radiation spectrum is $P_{\nu} \propto \nu$ for $\nu<\nu(\gamma)$ with a sharp cut off for $\nu>\nu(\gamma)$. An electron with an initial Lorentz factor $\gamma<\gamma_{\mathrm{b} 3}$ suffer energy loss via Coulomb loss, so that we obtain $P_{\nu} \propto t_{\mathrm{Clmb}}(\gamma) \propto \nu^{1 / 2}$ for $\nu<\nu(\gamma)$ with sharp cutoff for $\nu>\nu(\gamma)$.

To calculate the net spectrum from a power-law distribution of electrons, one needs to integrate over $\gamma$. There are three different cases, depending on $\gamma_{\text {min }}$, in which (A) $\gamma_{\mathrm{b} 3}<$ $\gamma_{\mathrm{b} 1}<\gamma_{\min }$, (B) $\gamma_{\mathrm{b} 3}<\gamma_{\min }<\gamma_{\mathrm{b} 1}$ and $(\mathrm{C}) \gamma_{\min }<\gamma_{\mathrm{b} 3}<\gamma_{\mathrm{b} 1}$. Below we provide additional details on these regimes.

- $\left(\right.$ A) $\gamma_{\mathrm{b} 3}<\gamma_{\mathrm{b} 1}<\gamma_{\min }$ (i.e., $\left.\nu_{\mathrm{b} 3}<\nu_{\mathrm{b} 1}<\nu_{\mathrm{m}}\right)$ :

In this case, all the electrons cool down to $\gamma_{\mathrm{b} 1}$. The spectral power is given by,

$\nu L_{\nu}= \begin{cases}L_{0}\left(\nu / \nu_{\mathrm{m}}\right)^{(2-p) / 2} & \left(\mathrm{~A} 1: \nu_{\mathrm{m}}<\nu\right) \\ L_{0}\left(\nu / \nu_{\mathrm{m}}\right)^{1 / 2} & \left(\mathrm{~A} 2: \nu_{\mathrm{b} 1}<\nu<\nu_{\mathrm{m}}\right) \\ L_{0}\left(\nu_{\mathrm{m}} / \nu_{\mathrm{b} 1}\right)^{3 / 2}\left(\nu / \nu_{\mathrm{m}}\right)^{2} & \left(\mathrm{~A} 3: \nu<\nu_{\mathrm{b} 1}\right)\end{cases}$

where,

$\nu_{\mathrm{m}}=\nu\left(\gamma_{\min }\right)$.

To determine the normalization constant, $L_{0}$, one can use the fact that electrons with $\gamma>\gamma_{\mathrm{b} 1}$ lose almost all their energy via IC emission, that is, the luminosity in the regime $\nu_{\mathrm{m}}<\nu$ can be written as (Loeb \& Waxman 2000; Keshet et al. 2003; Kushnir \& Waxman 2009),

$L_{\nu} d \nu=\gamma m_{e} c^{2} \dot{N}(\gamma) d \gamma$

Using Eqs. (10) and (18) and $d \nu / \nu=2 d \gamma / \gamma$ for $\nu \propto \gamma^{2}$, we obtain,

$$
\begin{aligned}
L_{0} & =\frac{3 \xi_{e} k T}{4 g(p)} \frac{\left(\Omega_{\mathrm{b}} / \Omega_{\mathrm{m}}\right) \dot{M}}{\mu m_{p}} \\
& =2.0 \times 10^{43} \frac{f_{\mathrm{T}} f_{\mathrm{acc}} \xi_{e,-2}}{g(p)} M_{14}^{5 / 3} w(z)^{5 / 6}(1+z)^{5 / 2} \mathrm{erg} \mathrm{s}
\end{aligned}
$$

where,

$$
\begin{gathered}
g(p)=\left\{\begin{array}{ll}
\ln \left(\gamma_{\max } / \gamma_{\min }\right) & (p=2) \\
\frac{1}{p-2}\left[1-\left(\gamma_{\min } / \gamma_{\max }\right)^{p-2}\right] & (p \neq 2)
\end{array} .\right. \\
\text { - }\left(\text { B) } \gamma_{\mathrm{b} 3}<\gamma_{\min }<\gamma_{\mathrm{b} 1}\left(\text { i.e., } \nu_{\mathrm{b} 3}<\nu_{\mathrm{m}}<\nu_{\mathrm{b} 1}\right):\right.
\end{gathered}
$$

1 This spectral slope is identical to the similar case considered in Sari, Piran, \& Naravan (1998) since both of the characteristic frequencies of the synchrotron and IC emissions are proportional to the square of the electron energy.
In this case, only those electrons with $\gamma>\gamma_{\mathrm{b} 1}$ can cool. We have,

$$
\nu L_{\nu}= \begin{cases}L_{0}\left(\nu / \nu_{\mathrm{m}}\right)^{(2-p) / 2} & \left(\mathrm{~B} 1: \nu_{\mathrm{b} 1}<\nu\right) \\ L_{0}\left(\nu_{\mathrm{m}} / \nu_{\mathrm{b} 1}\right)^{1 / 2}\left(\nu / \nu_{\mathrm{m}}\right)^{(3-p) / 2} & \left(\mathrm{~B} 2: \nu_{\mathrm{m}}<\nu<\nu_{\mathrm{b} 1}\right) \\ L_{0}\left(\nu_{\mathrm{m}} / \nu_{\mathrm{b} 1}\right)^{1 / 2}\left(\nu / \nu_{\mathrm{m}}\right)^{2} & \left(\mathrm{~B} 3: \nu<\nu_{\mathrm{m}}\right)\end{cases}
$$

where $L_{0}$ is given by Eq. (35).

\section{- $\left(\right.$ C) $\gamma_{\min }<\gamma_{\mathrm{b} 3}<\gamma_{\mathrm{b} 1}$ (i.e., $\left.\nu_{\mathrm{m}}<\nu_{\mathrm{b} 3}<\nu_{\mathrm{b} 1}\right)$ :}

In this case, electrons with $\gamma>\gamma_{\mathrm{b} 1}$ and $\gamma<\gamma_{\mathrm{b} 3}$ can cool. The electron spectrum has a break at $\gamma_{\mathrm{b} 3}$, below which it is well approximated with the stationary solution, $\propto \dot{N}(\gamma) t_{\mathrm{Clmb}}(\gamma) \propto \gamma^{1-p}$ (Sarazin 1999). Hence, we have $L_{\nu} \propto \nu^{(2-p) / 2}$ for $\nu_{\mathrm{m}}<\nu<\nu_{\mathrm{b} 3}$. Therefore, the luminosity is given by,

$\nu L_{\nu}= \begin{cases}L_{0}\left(\nu / \nu_{\mathrm{m}}\right)^{(2-p) / 2} & \left(\mathrm{C} 1: \nu_{\mathrm{b} 1}<\nu\right) \\ L_{0}\left(\nu_{\mathrm{m}} / \nu_{\mathrm{b} 1}\right)^{1 / 2}\left(\nu / \nu_{\mathrm{m}}\right)^{(3-p) / 2} & \left(\mathrm{C} 2: \nu_{\mathrm{b} 3}<\nu<\nu_{\mathrm{b} 1}\right) \\ L_{0}\left(\nu_{\mathrm{m}} / \nu_{\mathrm{b} 1}\right)^{1 / 2}\left(\nu_{\mathrm{m}} / \nu_{\mathrm{b} 3}\right)^{1 / 2}\left(\nu / \nu_{\mathrm{m}}\right)^{(4-p) / 2} & \left(\text { C3 }: \nu_{\mathrm{m}}<\nu<\nu_{\mathrm{b} 3}\right) \\ L_{0}\left(\nu_{\mathrm{m}} / \nu_{\mathrm{b} 1}\right)^{1 / 2}\left(\nu_{\mathrm{m}} / \nu_{\mathrm{b} 3}\right)^{1 / 2}\left(\nu / \nu_{\mathrm{m}}\right)^{3 / 2} & \left(\mathrm{C} 4: \nu<\nu_{\mathrm{m}}\right)\end{cases}$ where $L_{0}$ is given by Eq. (35).

\subsubsection{IC spectrum for $\nu_{\mathrm{b} 1}<\nu_{\mathrm{b} 2}<\nu_{\mathrm{b} 3}$}

In this case, the electrons suffer significant cooling throughout. Hence, an electron with an initial Lorentz factor $\gamma>\gamma_{\mathrm{b} 2}$ cools down through $\gamma_{\mathrm{b} 2}$ until which it produces $P_{\nu} \propto \nu^{-1 / 2}$, and further lose its energy to form $P_{\nu} \propto \nu^{1 / 2}$ below $\nu_{\mathrm{b} 2}$. The average spectrum from such electrons has a peak at $\nu_{\mathrm{b} 2}$. Similarly, an electron with an initial Lorentz factor $\gamma<\gamma_{\mathrm{b} 2}$ produces the average spectrum $P_{\nu} \propto \nu^{1 / 2}$ for $\nu<\nu(\gamma)$. In calculating the net spectrum for power-law electron distribution, there are two different cases in which (D) $\gamma_{\mathrm{b} 2}<\gamma_{\mathrm{m}}$ and (E) $\gamma_{\mathrm{m}}<\gamma_{\mathrm{b} 2}$. Below we describe them in detail.

\section{- $(\mathrm{D}) \gamma_{\mathrm{b} 2}<\gamma_{\min }$ (i.e., $\left.\nu_{\mathrm{b} 2}<\nu_{\mathrm{m}}\right)$ :}

Electrons with $\gamma>\gamma_{\text {min }}$ cools to make a spectrum $L_{\nu} \propto$ $\nu^{-p / 2}$ for $\nu>\nu_{\mathrm{m}}$, while electrons with $\gamma_{\mathrm{min}}$ forms the spectrum below $\nu_{\mathrm{m}}$. The luminosity is given by,

$$
\nu L_{\nu}= \begin{cases}L_{0}\left(\nu / \nu_{\mathrm{m}}\right)^{(2-p) / 2} & \left(\mathrm{D} 1: \nu_{\mathrm{m}}<\nu\right) \\ L_{0}\left(\nu / \nu_{\mathrm{m}}\right)^{1 / 2} & \left(\mathrm{D} 2: \nu_{\mathrm{b} 2}<\nu<\nu_{\mathrm{m}}\right) \\ L_{0}\left(\nu_{\mathrm{m}} / \nu_{\mathrm{b} 2}\right)\left(\nu / \nu_{\mathrm{m}}\right)^{3 / 2} & \left(\mathrm{D} 3: \nu<\nu_{\mathrm{b} 2}\right)\end{cases}
$$

where $L_{0}$ is given by Eq. (35).

- $(\mathrm{E}) \gamma_{\min }<\gamma_{\mathrm{b} 2}$ (i.e., $\left.\nu_{\mathrm{m}}<\nu_{\mathrm{b} 2}\right)$ :

In this case, electron distribution for $\gamma_{\min }<\gamma<\gamma_{\mathrm{b} 2}$ is proportional to $\dot{N}(\gamma) t_{\mathrm{Clmb}}(\gamma) \propto \gamma^{1-p}$. Hence, we have,

$\nu L_{\nu}= \begin{cases}L_{0}\left(\nu / \nu_{\mathrm{m}}\right)^{(2-p) / 2} & \left(\mathrm{E} 1: \nu_{\mathrm{b} 2}<\nu\right) \\ L_{0}\left(\nu_{\mathrm{m}} / \nu_{\mathrm{b} 2}\right)\left(\nu / \nu_{\mathrm{m}}\right)^{(4-p) / 2} & \left(\mathrm{E} 2: \nu_{\mathrm{m}}<\nu<\nu_{\mathrm{b} 2}\right) \\ L_{0}\left(\nu_{\mathrm{m}} / \nu_{\mathrm{b} 2}\right)\left(\nu / \nu_{\mathrm{m}}\right)^{3 / 2} & \left(\mathrm{E} 3: \nu<\nu_{\mathrm{m}}\right)\end{cases}$

where $L_{0}$ is given by Eq. (35).

\subsection{Observed surface brightness of IC emission}

The observed surface brightness $S_{\nu}$ (in units of erg $\mathrm{s}^{-1} \mathrm{~cm}^{-2} \mathrm{~Hz}^{-1} \mathrm{str}^{-1}$ ) of IC emission from a cluster at redshift $z$ is given by, 

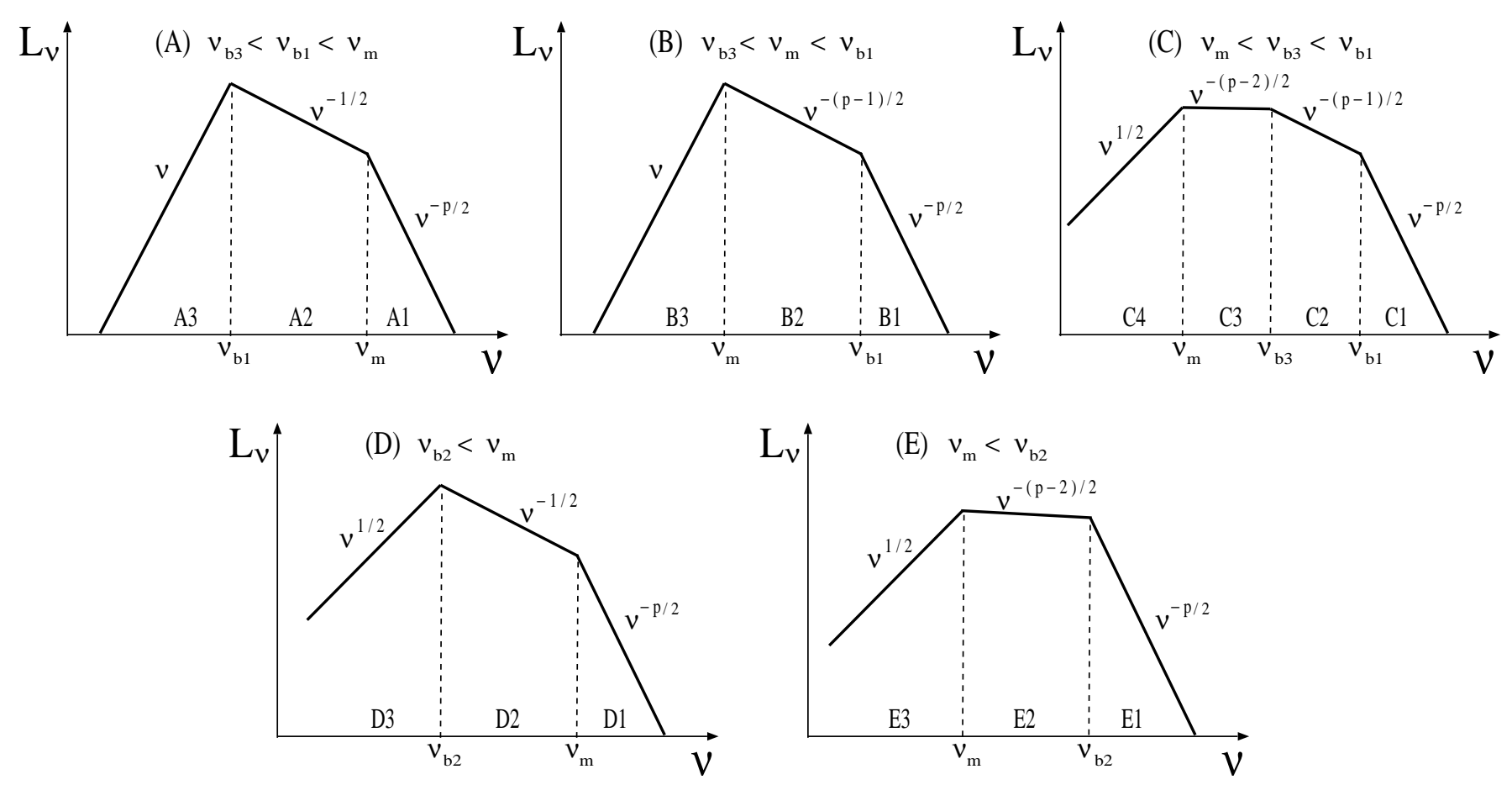

Figure 1. IC Spectrum from power-law distribution of relativistic electrons. Regimes (A) $\nu_{\mathrm{b} 3}<\nu_{\mathrm{b} 1}<\nu_{\mathrm{m}}$, (B) $\nu_{\mathrm{b} 3}<\nu_{\mathrm{m}}<\nu_{\mathrm{b} 1}$ and (C) $\nu_{\mathrm{m}}<\nu_{\mathrm{b} 3}<\nu_{\mathrm{b} 1}$ are for $\nu_{\mathrm{b} 3}<\nu_{\mathrm{b} 2}<\nu_{\mathrm{b} 1}$, while regimes (D) $\nu_{\mathrm{b} 2}<\nu_{\mathrm{m}}$ and (E) $\nu_{\mathrm{m}}<\nu_{\mathrm{b} 2}$ are for $\nu_{\mathrm{b} 1}<\nu_{\mathrm{b} 2}<\nu_{\mathrm{b} 3}$.

$S_{\nu}=\frac{(1+z) L_{\nu_{\mathrm{s}}}}{4 \pi d_{L}(z)^{2} \Omega_{\nu}}$

where $\nu_{\mathrm{s}}=(1+z) \nu, d_{L}(z)$ and $\Omega_{\nu}$ are the observing frequency translated into the cluster rest frame, the luminosity distance to the cluster and the solid angle of the extended emission on the sky, respectively.

The effective value of $\Omega_{\nu}$ can be estimated based on the observed brightness profile in the sky, which depends on the Lorentz factor of the electrons, $\gamma_{\nu}=(\nu / 2.05 \times$ $\left.10^{11} \mathrm{~Hz}\right)^{1 / 2}$, emitting IC photons with observed frequency $\nu$ [see Eq. (21)]. If the total cooling time of such electrons, $t_{\mathrm{cool}}\left(\gamma_{\nu}\right)=\left[t_{\mathrm{IC} / \mathrm{syn}}\left(\gamma_{\nu}\right)^{-1}+t_{\mathrm{Clmb}}\left(\gamma_{\nu}\right)^{-1}\right]^{-1}$, is much smaller than the dynamical time of the cluster $t_{\mathrm{dyn}}$, then the emission mainly originates from the region around the shock. The width of the emission region (in the radial direction) is given by the product of downstream flow velocity and the cooling time, $\delta \sim(1 / 3) V_{\mathrm{c}} t_{\mathrm{cool}}\left(\gamma_{\nu}\right)<r_{\mathrm{vir}}$, where a factor $1 / 3$ applies to the strong shock limit. The surface brightness profile has a rim-brightened shape. Because of projection, the observed apparent scale width, $W$, in the sky differs from $\delta$. The ratio $W / \delta$ depends on the uncertain radial profile of the electron distribution downstream of the shock, but is typically around 3 (Bamba et al. 2005a). Writing $W=f_{\mathrm{W}} \delta$, we obtain $\Omega_{\nu} \approx 2 \pi\left(f_{\mathrm{W}} / 3\right) r_{\mathrm{vir}} V_{\mathrm{c}} t_{\mathrm{cool}}\left(\gamma_{\nu}\right) / d_{\mathrm{A}}^{2}$, where $d_{\mathrm{A}}$ is the angular diameter distance to the cluster. On the other hand, if $t_{\text {cool }}\left(\gamma_{\nu}\right) \gg t_{\mathrm{dyn}}$, the cluster interior is filled with electrons with $\gamma_{\nu}$. Taking into account the projection effect, the surface brightness profile is center-filled. In this case, assuming the bright emission in the sky originates from inside the radius $r_{\text {vir }} / 2$, it occupies a solid angle $\Omega_{\nu} \approx(\pi / 4)\left(r_{\mathrm{vir}} / d_{\mathrm{A}}\right)^{2}$. Connecting both limits, we get

$$
\Omega_{\nu}=\frac{\pi}{4}\left(\frac{r_{\mathrm{vir}}}{d_{\mathrm{A}}}\right)^{2} \min \left\{1,8 \frac{f_{\mathrm{W}}}{3} \frac{t_{\mathrm{cool}}\left(\gamma_{\nu}\right)}{t_{\mathrm{dyn}}}\right\} .
$$

\section{RESULTS}

Based on the derivations in the previous sections, we can now calculate the observed surface brightness $S_{\nu}$ in the SDSS g-band $\left(\nu=6.3 \times 10^{14} \mathrm{~Hz}\right)$ as a function of redshift $z$ and a halo mass $M$. Other parameters are fixed at the fiducial values, $p=2.5, r_{4}=\xi_{\mathrm{B},-2}=\eta_{g}=\eta_{e,-5}=1, \xi_{e,-2}=5$, $f_{\text {acc }}=f_{\mathrm{T}}=0.5$ and $f_{\mathrm{W}}=3$. The parameter $\eta_{e}$ is highly uncertain and depends on upstream physical quantities such as magnetic field and gas temperature (e.g., Matsukiyo et al. 2011; Guo et al. 2014a, b). It can range from $\sim 10^{-7}$ to $\sim 10^{-4}$ (Kang et al. 2012). If $\eta_{e}$ is so large, then $\gamma_{\min }$ is less than unity [see Eq. (17)], so that our assumption of single power-law spectrum breaks down. Dependence of $\eta_{e}$ is easily found as seen in the following. The spectral index $p$ is also uncertain, however recent study of particle acceleration at cluster shocks suggest $p \approx 2.25-2.5$ (Kang \& Rvu 2011, 2013; Hong et al. 2014; Guo et al. 2014a). In the following, we also consider the cases of $p=2.0,2.3$ and 3.0 , as well as the fiducial case of $p=2.5$. The index $p$ is related to the shock compression ratio $r$. For first-order Fermi acceleration, $p=(r+2) /(r-1)$ in the test-particle limit (Blandford \& Ostriker 1978; Bell 1978). In this case, $r$ ranges between 2.5 and 4 for $2<p<3$. Nevertheless, we fix $r_{4}=(r / 4)=1$, which corresponds to the strong shock limit, because observed surface brightness does not depend on $r$ for the parameters of interest. All the equations in this section do not depend on $r$.

Figure 2 shows the surface brightness as a function of 
redshift $z$, for a fixed halo mass $M_{14}=\left(M / 10^{14} M_{\odot}\right)=3$. The red line describes the fiducial value of $p=2.5$, while the others are for different values of $p$ with the other parameters fixed at their fiducial values. Interestingly, the surface brightness increases with $z$. At low redshifts where the effects of cosmological expansion are negligible, the brightness is almost constant, $\approx 36 \mathrm{mag} \operatorname{arcsec}^{-2}$. As $z$ increases, the surface brightness becomes larger by $\approx 3$ mag until $z \approx 2$. For this redshift range, one can see from Figure 3 that $\nu_{\mathrm{b} 3}<\nu_{\mathrm{m}}<\nu_{\mathrm{s}}=(1+z) \nu<\nu_{\mathrm{b} 1}$, so that the spectrum is in the regime B2 [see Eq. (37)]. Thus, we find,

$$
\begin{aligned}
L_{\nu_{\mathrm{s}}}= & L_{0} \nu_{\mathrm{b} 1}^{-1 / 2} \nu_{\mathrm{m}}^{(p-2) / 2} \nu_{\mathrm{s}}^{(1-p) / 2} \\
\propto & f_{\mathrm{acc}} f_{\mathrm{T}}^{p-1} \xi_{e}^{p-1} \eta_{e}^{2-p} M_{14}^{(2 p+1) / 3} \\
& \quad \times w(z)^{(p-1) / 3}(1+z)^{3(p+1) / 2} \nu_{\mathrm{s}}^{(1-p) / 2}
\end{aligned}
$$

Since electron cooling is not significant, the solid angle of the emission is given by $\Omega_{\nu} \approx(\pi / 4)\left(r_{\mathrm{vir}} / d_{\mathrm{A}}\right)^{2}$, so that,

$$
\begin{aligned}
S_{\nu} \propto & r_{\mathrm{vir}}^{-2}(1+z)^{-3} L_{\nu_{\mathrm{s}}} \\
\propto & f_{\mathrm{acc}} f_{\mathrm{T}}^{p-1} \xi_{e}^{p-1} \eta_{e}^{2-p} M_{14}^{(2 p-1) / 3} \\
& \quad \times w(z)^{(p+1) / 3}(1+z)^{p+1} \nu^{(1-p) / 2} .
\end{aligned}
$$

Since $w(z)$ is only weakly dependent on $z$, the surface brightness $S_{\nu}$ increases with $z$ following the scaling $(1+z)^{p+1}$.

The regime B2 ends when $\nu_{\mathrm{s}}$ becomes larger than $\nu_{\mathrm{b} 1}$. This crossing occurs at $z \approx 2$ for our fiducial parameters. Thereafter, the spectrum is in regime B1. When $z$ further increases, the spectrum enters into regime A1, subsequently followed by the regime D1. In these regimes, the luminosity is given by the same form [see Eqs. (32), (37) and (39)],

$$
\begin{aligned}
L_{\nu_{\mathrm{s}}}= & L_{0} \nu_{\mathrm{m}}^{(p-2) / 2} \nu_{\mathrm{s}}^{-p / 2} \\
\propto & f_{\mathrm{acc}} f_{\mathrm{T}}^{p-1} \xi_{e}^{p-1} \eta_{e}^{2-p} M_{14}^{(2 p+1) / 3} \\
& \quad \times w(z)^{(2 p+1) / 6}(1+z)^{(3 p-1) / 2} \nu_{\mathrm{s}}^{-p / 2},
\end{aligned}
$$

and the solid angle is still given by $\Omega_{\nu} \approx(\pi / 4)\left(r_{\mathrm{vir}} / d_{\mathrm{A}}\right)^{2}$, so that we have,

$$
\begin{aligned}
S_{\nu} \propto \quad f_{\mathrm{acc}} f_{\mathrm{T}}^{p-1} \xi_{e}^{p-1} \eta_{e}^{2-p} M_{14}^{(2 p-1) / 3} & \\
& \times w(z)^{(2 p+5) / 6}(1+z)^{(2 p-3) / 2} \nu^{-p / 2} .
\end{aligned}
$$

For our fiducial parameters, Eq. (46) describes the scaling if $2<z<5.1$. The flux increases by a factor of about 3 in this redshift range. Note that the emergence of these regimes originates from rapid decreasing of $\nu_{\mathrm{b} 1} \propto(1+z)^{-4}$ for $z>1$. As a result, the blue-shifted observing frequency $\nu_{\mathrm{s}}=(1+z) \nu$ becomes larger than any other characteristic frequencies, $\nu_{\mathrm{m}}$ and $\nu_{\mathrm{b} i}(i=1,2,3)$, around $z_{\mathrm{eq}}$. As $z$ further increases, $\nu_{\mathrm{m}} \propto(1+z)^{2}$ together with $\nu_{\mathrm{b} 3} \propto(1+z)^{4}$ become large, finally exceeding $\nu_{\mathrm{s}}$, so that the spectrum enters the regime D2. There the electron cooling is so significant that the observed brightness profile is rim-brightened shape, so that $\Omega_{\nu}$ is small and $S_{\nu}$ shows rapid increase for $z \gtrsim 6$. However, the abundance of clusters at these high redshifts is extremely small (Barkana \& Loeb 2001; Watson et al. 2013).

Although the parameter dependence is somewhat complicated, one can see that overall behavior is not so different from the fiducial parameter set; the brightness varies typically by up to $\approx 2-3 \mathrm{mag}$ if one of parameters is changed with others fixed. Figure 2 shows lines for the cases of $p=2.0,2.3$ and 3.0 with other parameters fixed as fiducial. The larger the $p$, the brighter the surface emission. The de-

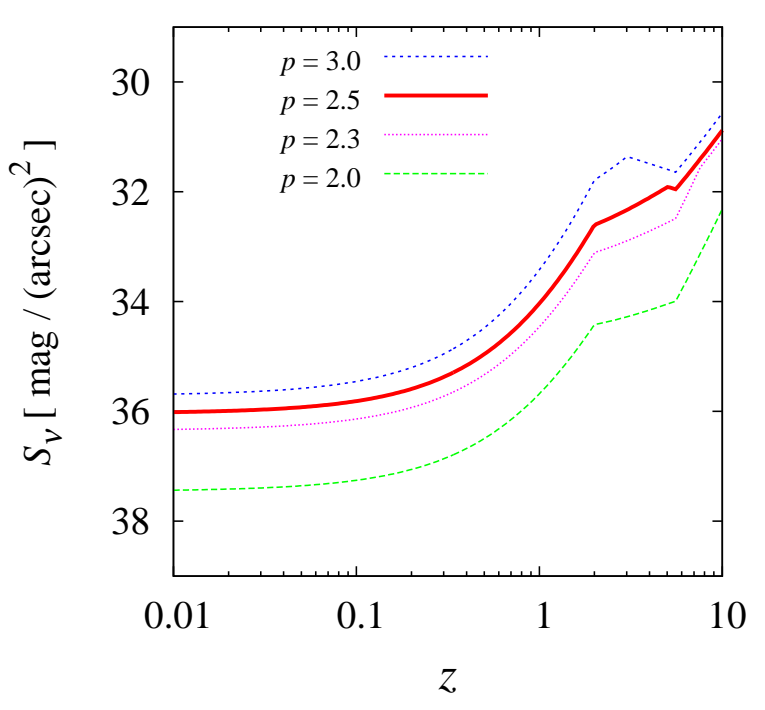

Figure 2. Observed SDSS g-band surface brightness of IC emission from a cluster with $M_{14}=3$ (in units of mag $\operatorname{arcsec}^{-2}$ ) as a function of cluster redshift $z$. The red line is for fiducial parameters $\left(p=2.5, r_{4}=\xi_{\mathrm{B},-2}=\eta_{g}=\eta_{e,-5}=1, \xi_{e,-2}=5\right.$, $f_{\text {acc }}=f_{\mathrm{T}}=0.5$ and $f_{\mathrm{W}}=3$ ), while the green, purple and blue lines are for $p=2.0,2.3$ and 3.0, respectively.

pendence on other parameters can be found from Eqs. (44) and (46).

Figure 4 depicts the surface brightness as a function of a halo mass $M$ for fixed cluster redshifts assuming the fiducial parameter values. As seen in Fig. 2 the redshift dependence is small when $z \lesssim 0.1$. There, the surface brightness peaks at $M_{14} \approx 30$, below which the spectrum is in regime $\mathrm{B} 2$ for $0.55 \lesssim M_{14} \lesssim 30$ and in regime $\mathrm{C} 2$ for $M_{14} \lesssim 0.55$. Functional forms of the brightness for $\mathrm{B} 2$ and $\mathrm{C} 2$ are given by Eq. (44), with $S_{\nu} \propto M_{14}^{(2 p-1) / 3}$. For $M_{14} \gtrsim 30$ the spectrum is in regime $\mathrm{B} 3$, at which we have,

$S_{\nu} \propto f_{\mathrm{acc}} f_{\mathrm{T}}^{-2} \xi_{e}^{-2} \eta_{e}^{3} M_{14}^{-1} w(z) \nu$,

so that the brightness decreases with $M$. For $z=0.5$, the peak shifts to lower masses, and larger values. Note that Eq. (47) implies that the brightness in regime B3 very weakly depends on $z$. The behavior for $z=1.3$ is the same as for lower redshifts as long as $M_{14}<33$, above which, however, the spectrum is in the regime A3 and the surface brightness again increases with $M$ and $z$ according to the scaling,

$S_{\nu} \propto f_{\text {acc }} \eta_{e} M_{14}^{1 / 3} w(z)^{1 / 3}(1+z)^{7} \nu$.

For higher redshifts $z=2.0(z=4.0)$, the spectrum enters regimes $\mathrm{C} 2, \mathrm{~B} 2$ and $\mathrm{B} 1$ (E1 and $\mathrm{D} 1)$ in turn towards higher 


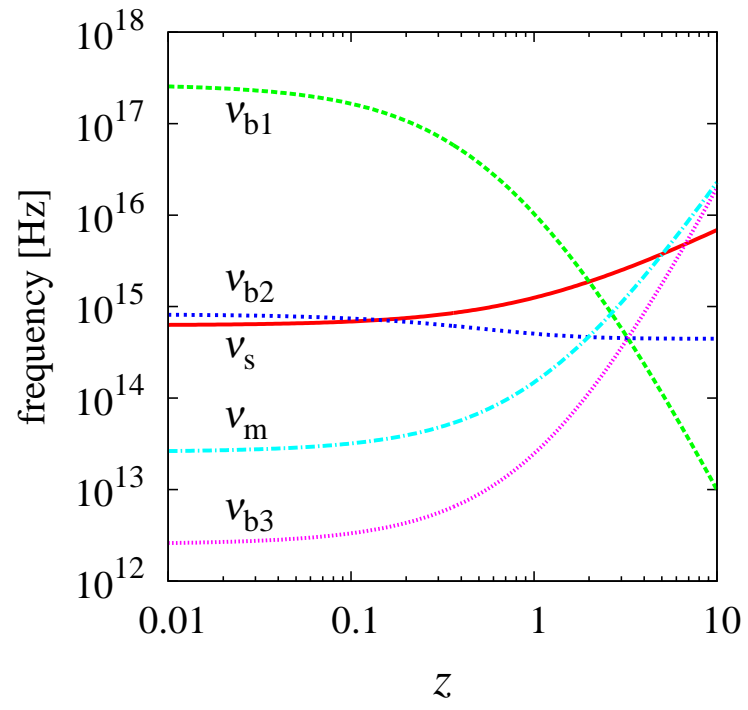

Figure 3. Characteristic frequencies, as measured in the rest frame of a cluster, for our fiducial parameter values with a halo mass $M_{14}=3$, as a function of redshift $z$. The green, blue, purple and light blue lines indicates $\nu_{\mathrm{b} 1}, \nu_{\mathrm{b} 2}, \nu_{\mathrm{b} 3}$ and $\nu_{\mathrm{m}}$, respectively. The red line represents $\nu_{\mathrm{s}}=(1+z) \nu$, which is the observed frequency blue-shifted to the rest frame of the cluster.

$M$. The brightness breaks at $M_{14}=8.5$ and 3.9 for $z=2.0$ and 4.0, respectively. After the break, the brightness still increases with $M$ according to the scaling,

$S_{\nu} \propto f_{\mathrm{acc}} \eta_{e} M_{14}^{1 / 3} w(z)^{7 / 6}(1+z)^{-1 / 2} \nu^{-1 / 2}$.

In summary, the surface brightness follows simple scaling behavior, $S_{\nu} \propto M^{(2 p-1) / 3}$, for the typical range of expected parameters.

\section{DISCUSSION}

Using a simple analytical model, we have calculated IC emission in the SDSS g-band from relativistic electrons accelerated in galaxy clusters, taking into account the effects of Coulomb, synchrotron, and IC energy loss of the emitting electrons. For our fiducial parameters, at $z \lesssim 2$ and $M \lesssim$ $10^{15} M_{\odot}$, the spectrum is in the regime B2 or $\mathrm{C} 2$, in which $S_{\nu} \propto M^{(2 p-1) / 3}(1+z)^{p+1} \nu^{(1-p) / 2}$, where $p$ is the power-law index of electron distribution (see Fig. 4). If the value of $p$ is inferred from radio synchrotron emission, one can predict the spectral index of the optical IC emission. In this paper, we have not taken into account the possibility of reacceleration in the downstream turbulence, which enhances the abundance of relativistic electrons (Schlickeiser et al. 1987;

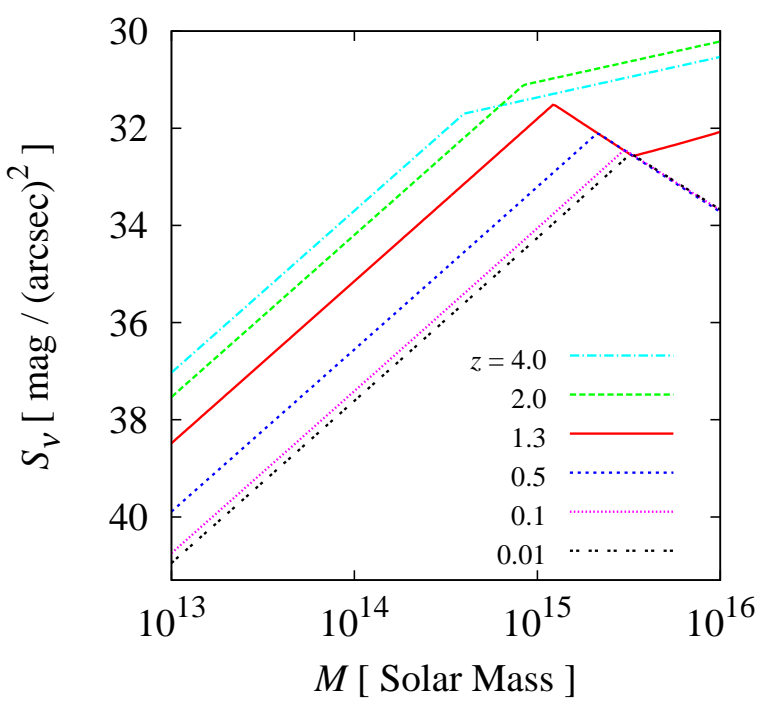

Figure 4. Observed SDSS g-band surface brightness (in units of mag $\operatorname{arcsec}^{-2}$ ) as a function of a halo mass $M$ for our fiducial parameters $\left(p=2.5, r_{4}=\xi_{\mathrm{B},-2}=\eta_{g}=\eta_{e,-5}=1, \xi_{e,-2}=5\right.$, $f_{\mathrm{acc}}=f_{\mathrm{T}}=0.5$ and $\left.f_{\mathrm{W}}=3\right)$. Different lines correspond to different redshifts $z$ of the cluster.

Brunetti et al. 2001; Petrosian 2001; Fuiita et al. 2003), resulting in brighter IC emission. We also assume a spherical, virialized shock as a result of smooth accretion. If the cluster is not dynamically relaxed and is still being formed, e.g. as a result of a merger of two sub-components, then the shock structure would be different and turbulent energy would be enhanced. In this case, we expect brighter IC emission due to more efficient reacceleration. Hence, our estimate for the IC flux and surface brightness should be regarded as a conservative lower limit.

It would be natural to assume that electron acceleration occurs at the virial shocks. In merging clusters, radio relics have been detected as possible signatures of the merger shocks, although clear evidence for it had not been identified as of yet. Observations at other wavelengths would be helpful for shock identification. Proton acceleration at the virial shocks could also occur, although we have only a few observational implications of it (e.g., Fuiita et al. 2013). Observations of optical IC emission may have the advantage of enabling the identification of shocks, because the angular resolution of the optical telescopes is generally much better than hard X-ray or gamma-ray telescopes. According to the theory of diffusive shock acceleration, electrons responsible for the optical IC emission cannot penetrate far upstream relative to the shock front. Hence, the IC emissivity 
is expected to have a steep rise across the shock front. The detection of such a sharp jump in the IC emission would flag the position of the shock front, and provide evidence for particle acceleration there. In our spherical model, however, IC-emitting electrons are not rapidly cooling in most cases, resulting in center-filled shape of the brightness profile on the sky. Thus, the profile may not have a sharp rise in projection. Possible exceptions might be expected for more realistic, non-spherical cases, where sharp feature could be found on the sky at the location of shocks. Detailed morphological studies based on numerical simulations, like the case of radio relics (e.g., Skillman et al. 2013; Hong et al. 2015), are needed in this case and go beyond the scope of this paper.

Our model predicts the surface brightness of IC emission from several massive clusters, as summarized in Table 1. Larger fluxes are expected for larger $M$ and $z$ if the spectrum is in regime B2 or C2 [see Eq. (44)]. The predicted brightness ranges between $\approx 32$ and $35 \mathrm{mag} \operatorname{arcsec}^{-2}$. Such ultralow surface brightnesses should be detectable with the recently developed Dragonfly Telephoto Array (Abraham \& van Dokkum 2014). More detailed prospects for the observation of individual clusters will be given elsewhere.

Our model also predicts the color of the IC emission, which is bluer than starlight. If we assume a power-law form of the IC emission, as $S_{\nu} \propto \nu^{-\alpha}$, then the color $g-r$ is calculated as

$g-r=\frac{5}{2} \alpha \log _{10}\left(\nu_{g} / \nu_{r}\right)=0.29 \alpha$,

where $\nu_{g}=6.3 \times 10^{14} \mathrm{~Hz}$ and $\nu_{r}=4.8 \times 10^{14} \mathrm{~Hz}$ are SDSS $\mathrm{g}$-band and r-band frequencies, respectively. In the case of spectral regime $\mathrm{B} 2$ or $\mathrm{C} 2$ (i.e., $\alpha=(p-1) / 2$ ), we obtain $g-r=0.19$ for $p=2.3$. If the spectral regime is in B3 (i.e., $\alpha=-1$ ), then $g-r=-0.29$. These values are distinguishable from stellar components, such as diffuse faint emission of brightest cluster galaxies (BCG), satellite galaxies, and intracluster light (ICL), which typically shows $g-r \gtrsim 0.7$ (e.g., Montes \& Truillo 2014).

Gamma-ray and hard X-ray observations have given upper limits, which constrain our model parameters. Figures 5 and 6 show that for our fiducial parameters (namely $p=2.5$ ), both IC gamma-ray and X-ray fluxes for nearby $(z \ll 0.1)$, massive $\left(M_{14}=30\right)$ clusters exceed current observational upper limits, which are $N(0.2-100 \mathrm{GeV}) \lesssim 10^{-9}$ $10^{-8}$ photons $\mathrm{cm}^{-2} \mathrm{~s}^{-1}$ for gamma-rays Ackermann et al. 2010) and $F(12-60 \mathrm{keV}) \lesssim 10^{-11}-10^{-10} \mathrm{erg} \mathrm{cm}^{-2} \mathrm{~s}^{-1}$ for X-rays (Ota et al. 2014). Although the case with $p \lesssim 2.5$ is unlikely for such nearby massive clusters, our model predictions can be lower than these current observational upper limits for less massive $\left(M_{14} \lesssim 10\right)$, higher redshift $(z \gtrsim 0.1)$ or steeper electron index $(p \gtrsim 2.8) \sqrt{2}$. Note that we conservatively adopt $p=2.5$ in this paper, but that higher values of $p$ as implied by the gamma-ray and X-ray limits for low red-

2 Some X-ray observations for specific clusters have provided tight upper limits, such as $F(12-60 \mathrm{keV})<5 \times$ $10^{-12} \mathrm{erg} \mathrm{cm}^{-2} \mathrm{~s}^{-1}$ (Nishino et al. 2010) for Perseus $(z=0.018$ and $\left.M_{200}=1.2 \times 10^{15} M_{\odot}\right)$, while our model overpredicts X-ray flux by a factor of 3 larger even for $p=3.0$. In such cases, smaller value of $\xi_{e}(\lesssim 0.01)$ may be required.

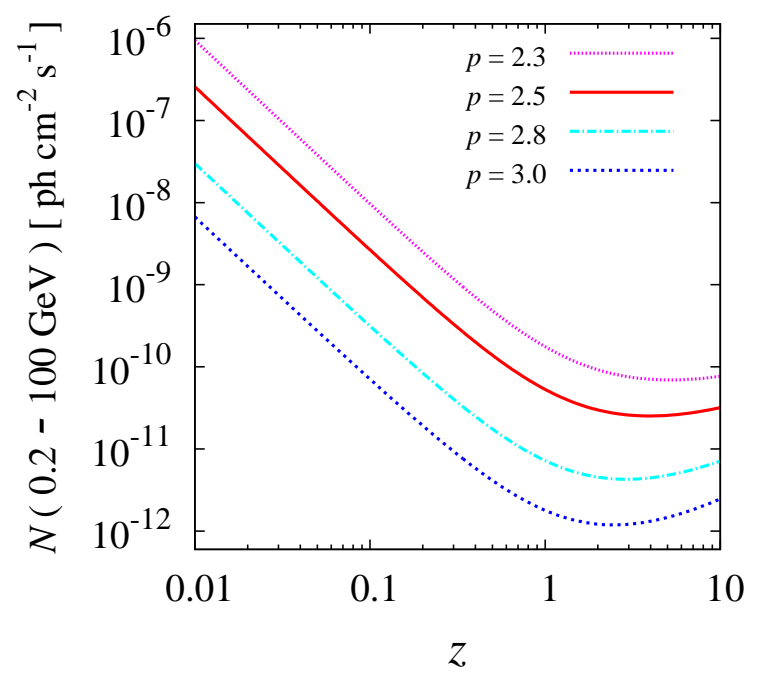

Figure 5. Observed IC gamma-ray flux $N(0.2-100 \mathrm{GeV})$, in units of photons $\mathrm{cm}^{-2} \mathrm{~s}^{-1}$, as a function of redshift $z$ for most massive $\left(M_{14}=30\right)$ clusters. The red line is for fiducial parameters $\left(p=2.5, r_{4}=\xi_{\mathrm{B},-2}=\eta_{g}=\eta_{e,-5}=1\right.$, $\xi_{e,-2}=5, f_{\mathrm{acc}}=f_{\mathrm{T}}=0.5$ and $f_{\mathrm{W}}=3$ ), while the purple, light blue and blue lines are for $p=2.3,2.8$ and 3.0, respectively. Mass dependence of the gamma-ray flux is given by Eq. [45], as $N(0.2-100 \mathrm{GeV}) \propto M_{14}^{(2 p+1) / 3}$, because observed frequency blue-shifted to the rest frame, $\nu_{\mathrm{s}}$, is always larger than any other characteristic frequencies, $\nu_{\mathrm{b} 1}, \nu_{\mathrm{b} 2}, \nu_{\mathrm{b} 3}$ and $\nu_{\mathrm{m}}$. Observed upper limits for specific clusters lie $N(0.2-100 \mathrm{GeV}) \lesssim 10^{-9}$ $10^{-8}$ photons $\mathrm{cm}^{-2} \mathrm{~s}^{-1}$ Ackermann et al. 2010).

shift clusters would make our predicted optical IC flux somewhat higher (see Fig. 2). Another independent way to have lower gamma-ray flux is to adopt unusually large $\eta_{g} \gg 10^{2}$. In this case, $\gamma_{\max }$ is small enough to give spectral cutoff of IC emission at energy below the Fermi band.

The IC emission from the virial shock of clusters could be enhanced due to relativistic electrons produced in supernovae that gradually diffuse out of the cluster. The latter contribution depends on the star formation history and the diffusion time of relativistic electrons within the cluster. The diffusion time of electrons depends on the unknown configuration of magnetic fields. If the fields are radially aligned in the outer envelope of clusters (as expected from radial infall or the magneto-thermal instability; see e.g., Parrish et al. 2012), then the diffusion time there would be of order the light crossing time of the outer parts of the cluster, i.e. millions of years. The Fermi satellite has placed tight limits on this contribution in cluster cores based 
Table 1. Predicted surface brightness in SDSS g-band for specific clusters.

\begin{tabular}{|c|c|c|c|c|c|c|}
\hline \multirow[t]{2}{*}{ Name } & \multirow[t]{2}{*}{$z$} & \multirow[t]{2}{*}{$\begin{array}{r}M_{\odot}^{a} \\
{\left[10^{14} M_{\odot}\right]}\end{array}$} & \multicolumn{3}{|c|}{$\begin{array}{l}\text { Surface brightness } \\
\\
{\left[\mathrm{mag} \mathrm{arcsec}^{-2}\right]}\end{array}$} & \multirow[t]{2}{*}{ Reference } \\
\hline & & & $p=2.0$ & $p=2.5$ & $p=3.0$ & \\
\hline IDCS J1426.5+3508 & 1.75 & 5.3 & $34.1(\mathrm{C} 2)$ & 32.1 (B2) & $31.2(\mathrm{~B} 3)$ & Stanford et al. (2012) \\
\hline SPT-CL J2106-5844 & 1.132 & 9.8 & $34.2(\mathrm{~B} 2)$ & 32.1 (B2) & 31.8 (B3) & Williamson et al. (2011) \\
\hline ACT-CL J0102-4915 & 0.870 & 22.3 & 33.7 (B2) & 32.1 (B3) & 32.7 (B3) & Menanteau et al. (2012) \\
\hline SPT-CL J2344-4243 & 0.596 & 25.0 & $34.0(\mathrm{~B} 2)$ & $32.3(\mathrm{~B} 3)$ & $32.8(\mathrm{~B} 3)$ & McDonald et al. (2012) \\
\hline MS 1054-0321 & 0.83 & 12 & $34.4(\mathrm{~B} 2)$ & $32.3(\mathrm{~B} 2)$ & $32.0(\mathrm{~B} 3)$ & Jee et al. (2005) \\
\hline SPT-CL J0658-5556 & 0.296 & 31.2 & $34.3(\mathrm{~B} 2)$ & 32.5 (B3) & 33.1 (B3) & Williamson et al. (2011) \\
\hline XDCP J0044.0-2033 & 1.579 & 4.4 & $34.5(\mathrm{C} 2)$ & $32.6(\mathrm{~B} 2)$ & $31.7(\mathrm{~B} 2)$ & Tozzi et al. (2015) \\
\hline SPT-CL J2337-5942 & 0.775 & 10.5 & $34.7(\mathrm{~B} 2)$ & $32.6(\mathrm{~B} 2)$ & 31.9 (B3) & Williamson et al. (2011) \\
\hline Coma & 0.0232 & 27.8 & $35.0(\mathrm{~B} 2)$ & $32.7(\mathrm{~B} 2)$ & $32.9(\mathrm{~B} 3)$ & Kubo et al. (2007) \\
\hline MACS J1206.2-0847 & 0.44 & 14.1 & $34.9(\mathrm{~B} 2)$ & $32.8(\mathrm{~B} 2)$ & $32.2(\mathrm{~B} 3)$ & Presotto et al. (2014) \\
\hline Abell 2390 & 0.228 & 18 & $35.1(\mathrm{~B} 2)$ & $32.9(\mathrm{~B} 2)$ & 32.5 (B3) & Carlberg et al. (1996) \\
\hline XLSSU J021744.1-034536 & 1.91 & $\approx 2^{c}$ & $35.0(\mathrm{C} 2)$ & $33.3(\mathrm{~B} 2)$ & $32.6(\mathrm{~B} 2)$ & Mantz et al. (2014) \\
\hline Abell 2744 & 0.3064 & 70 & $33.4(\mathrm{~B} 2)$ & 33.3 (B3) & 33.9 (B3) & Montes \& Truiillo (2014) \\
\hline
\end{tabular}

\footnotetext{
$a$ Values of $M_{200}$ are taken from references.

$b$ For fiducial parameters other than $p$. Spectral regime is also shown in parentheses.

$c$ Since only $M_{500}$ is given in Mantz et al. (2014), we estimate $M_{200}$ assuming isothermal density distribution as $M_{200}=1.58 M_{500}$.
}

on the lack of gamma-ray emission at $0.2-100 \mathrm{GeV}$ there (Ackermann et al. 2010, 2014, see also Vazza et al. 2015).

Diffuse optical emission from Thomson scattering of starlight could be comparable to the IC emission. We roughly estimate the flux of the scattered light emission as,

$\nu F_{\nu}^{(\mathrm{sc})} \sim \frac{L_{*} \tau_{\mathrm{T}}}{4 \pi d_{L}(z)^{2}}$

where $L_{*}$ is the bolometric stellar luminosity and $\tau_{\mathrm{T}}$ is the optical depth for the Thomson scattering. The typical massto-light ratio within the virial radius $r_{\mathrm{vir}}$ is given by $M / L \approx$ $2.5 \times 10^{2} M_{\odot} / L_{\odot}$ (e.g., Sheldon et al. 2009; Holland et al. $2015)$, so that $L_{*} \approx 1.6 \times 10^{45} M_{14} \mathrm{erg} \mathrm{s}^{-1}$. The optical depth $\tau_{\mathrm{T}}$ can be estimated as,

$$
\begin{aligned}
\tau_{\mathrm{T}} & \sim n_{e} \sigma_{\mathrm{T}} r_{\mathrm{vir}} \\
& \sim 3.3 \times 10^{-4} r_{4} f_{\mathrm{acc}} M_{14}^{1 / 3} w(z)^{2 / 3}(1+z)^{2} .
\end{aligned}
$$

Note that this estimate may be upper limit since we implicitly assume that most of the energy of the starlight is contained in the SDSS g-band in the observer frame. In Figure 7 we show $F_{\nu}^{(\mathrm{sc})}$ as a function of redshift $z$ (green lines), comparing with the IC flux (red lines). One can see that the IC emission dominates if $M_{14} \gtrsim 3$. Since the spectrum of the scattered light is very different from that of the IC emission, color measurements can be used to distinguish between them.

The BCG and ICL could also constitute a diffuse background. The ICL emission has been calculated based on the cosmological simulation (e.g., Rudick et al. 2011; Laporte et al. 2013; Cui et al. 2014). According to the recent result by Cui et al. (2014), the ICL brightness could be around 30 mag $\operatorname{arcsec}^{-2}$ at $r_{\text {vir }}$ for the most massive clusters. On the other hand, many observations measure the brightness profile of the BCG + ICL only in the interior of clusters due to its faintness (e.g., Presotto et al. 2014; DeMaio et al. 2015). Extrapolating linearly the observed radial profile of BCG+ICL of a cluster MACS J1206.2-0847 (Presotto et al.
2014) to its virial radius of $r_{\mathrm{vir}} \sim 2.3 \mathrm{Mpc}$ yields emission that is dimmer than $\sim 35 \mathrm{mag} \operatorname{arcsec}^{-2}$, so that IC emission is brighter for this cluster (see Table 1). Even if BCG+ICL has comparable brightness to the IC emission, their color difference can be used to separate them from each other.

IC emission also lies in infrared bands; however, the flux is rather small because typically $\nu_{\mathrm{s}}\left\langle\nu_{\mathrm{m}}\right.$ (i.e., it is in regime B3). Furthermore, mid and far-infrared bands may be dominated by dust emission (Yamada \& Kitavama 2005; Kitayama et al. 2009).

Finally, we remark on IC emission as a possible background emission for other purposes. For example, as already discussed above, the BCG+ICL brightness around the virial radius $r_{\text {vir }}$ could be comparable to the IC emission. The surface brightness of dwarf galaxies (e.g., Herrmann et al. 2013) is also in some cases less than $32 \mathrm{mag} \operatorname{arcsec}^{-2}$ at the periphery of the galaxies. The IC emission could confuse or disguise these different emissions from member galaxies in massive clusters.

\section{ACKNOWLEDGMENTS}

We thank Roberto Abraham, Yutaka Fujita, Hyesung Kang, Yutaka Ohira, Kouji Ohta, Dongsu Ryu, Lorenzo Sironi, Shuta Tanaka, Makoto Uemura and Pieter van Dokkum, for valuable comments. We also thank the anonymous referee for valuable comments to improve the paper. This work was supported in part by grant-in-aid from the Ministry of Education, Culture, Sports, Science, and Technology (MEXT) of Japan, No. 15K05088 (R. Y.) and NSF grant AST-1312034 (A. L.). R. Y. also thank ISSI (Bern) for support of the team "Physics of the Injection of Particle Acceleration at Astrophysical, Heliospheric, and Laboratory Collisionless Shocks". 


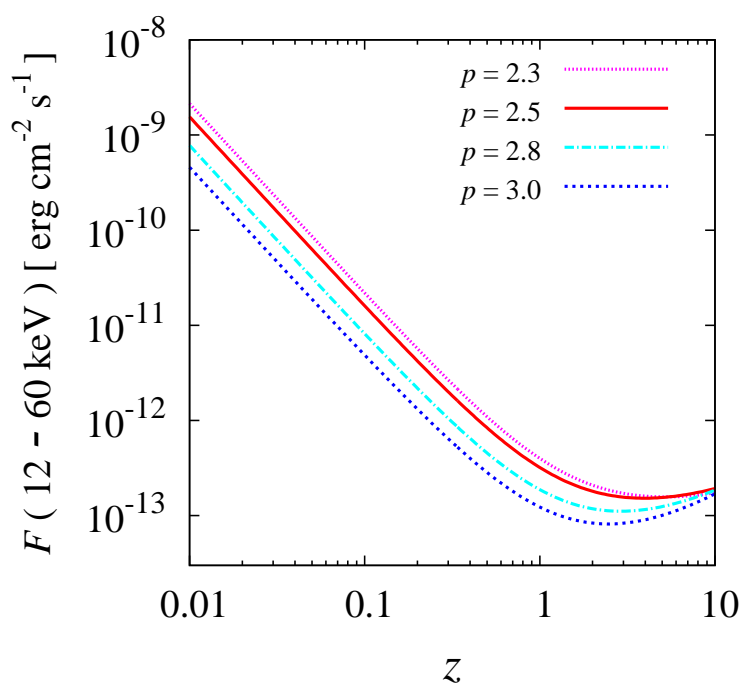

Figure 6. Observed IC X-ray flux $F(12-60 \mathrm{keV})$, in units of erg $\mathrm{cm}^{-2} \mathrm{~s}^{-1}$, as a function of redshift $z$ for most massive $\left(M_{14}=30\right)$ clusters. The red line is for fiducial parameters $(p=$ $2.5, r_{4}=\xi_{\mathrm{B},-2}=\eta_{g}=\eta_{e,-5}=1, \xi_{e,-2}=5, f_{\mathrm{acc}}=f_{\mathrm{T}}=0.5$ and $f_{\mathrm{W}}=3$ ), while the purple, light blue and blue lines are for $p=2.3,2.8$ and 3.0, respectively. Mass dependence of the gammaray flux is given by Eq. 45), as $F(12-60 \mathrm{keV}) \propto M_{14}^{(2 p+1) / 3}$, because observed frequency blue-shifted to the rest frame, $\nu_{\mathrm{s}}$, is always larger than any other characteristic frequencies, $\nu_{\mathrm{b} 1}$, $\nu_{\mathrm{b} 2}, \nu_{\mathrm{b} 3}$ and $\nu_{\mathrm{m}}$. Observed upper limits for specific clusters lie $F(12-60 \mathrm{keV}) \lesssim 10^{-11}-10^{-10} \mathrm{erg} \mathrm{cm}^{-2} \mathrm{~s}^{-1}$ (Ota et al. 2014).

\section{REFERENCES}

Abraham, R. G., \& van Dokkum, P. G. 2014, PASP, 126, 55

Ackermann, M., Ajello, M., Allafort, A., et al. 2010, ApJL, 717, L71

Ackermann, M., Ajello, M., Albert, A., et al. 2014, ApJ, 787, 18

Ade, P. A. R. et al. (Planck Collaboration) 2015, arXiv:1502.01589

Bamba, A. et al. 2003, ApJ, 589, 827

Bamba, A. et al. 2005a, ApJ, 621, 793

Bamba, A. et al. 2005b, ApJ, 632, 294

Bartels, R., Zandanel, F., \& Ando, S. 2015, A\&A in press (arXiv:1501.06940)

Bell, A. R. 1978, MNRAS, 182, 147

Barkana, R., \& Loeb, A. 2001, Phys. Rep., 349, 125

Blandford, R. D., \& Ostriker, J. P. 1978, ApJ, 221, L29

Blasi, P., \& Colafrancesco, S. 1999, Astroparticle Physics, 12,169

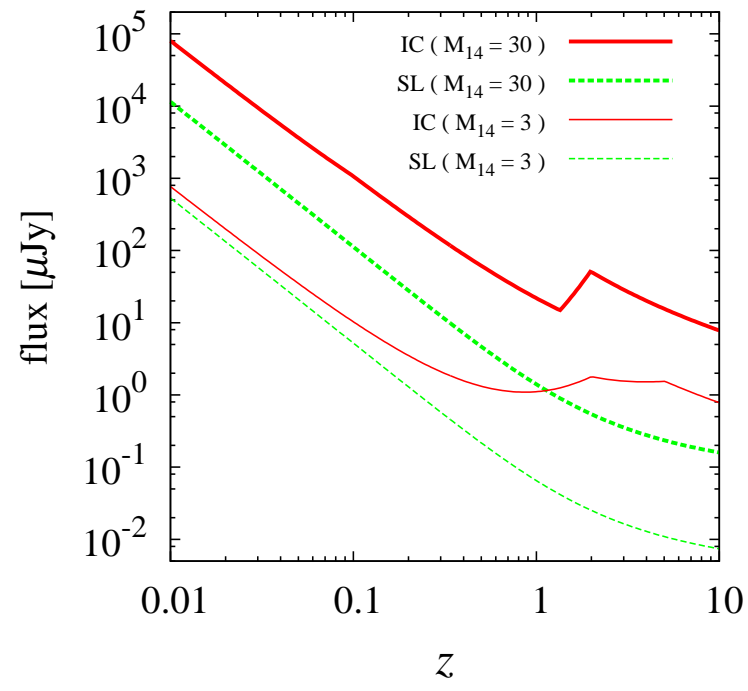

Figure 7. Observed SDSS g-band flux (in units of $\mu \mathrm{Jy}$ ) of IC emission (red lines) and the Thomson scattered starlight (green lines) as a function of redshift $z$. Thick and thin lines are for a halo mass $M_{14}=30$ and $M_{14}=3$, respectively. In calculating the IC flux, we adopt fiducial parameters $\left(p=2.5, r_{4}=\xi_{\mathrm{B},-2}=\right.$ $\eta_{g}=\eta_{e,-5}=1, \xi_{e,-2}=5, f_{\mathrm{acc}}=f_{\mathrm{T}}=0.5$ and $\left.f_{\mathrm{W}}=3\right)$.

Blumenthal, G. R., \& Gould, R. J. 1970, Reviews of Modern Physics, 42, 237

Brunetti, G., Setti, G., Feretti, L., \& Giovannini, G. 2001, MNRAS, 320, 365

Brunetti, G., \& Jones, T. W. 2014, Int. J. of Mod. Phys. D, 23, 1430007

Bryan, G. L., \& Norman, M. L. 1998, ApJ, 495, 80

Carlberg, R. G., Yee, H. K. C., Ellingson, E., et al. 1996, ApJ, 462, 32

Cui, W., Murante, G., Monaco, P., et al. 2014, MNRAS, 437,816

DeMaio, T., Gonzalez, A. H., Zabludoff, A., Zaritsky, D., \& Bradač, M. 2015, MNRAS, 448, 1162

Drury, L. O'C., 1983, Rep. Prog. Phys., 46, 973

Fixsen, D. J. 2009, ApJ, 707, 916

Fujita, Y., \& Kato, T. N. 2005, MNRAS, 364, 247

Fujita, Y., Ohira, Y., \& Yamazaki, R. 2013, ApJL, 767, L4

Fujita, Y., \& Sarazin, C. L. 2001, ApJ, 563, 660

Fujita, Y., Takizawa, M., \& Sarazin, C. L. 2003, ApJ, 584, 190

Gastaldello, F., Wik, D. R., Molendi, S., et al. 2015, ApJ, 800, 139

Guo, X., Sironi, L., \& Narayan, R. 2014a, ApJ, 794, 153

Guo, X., Sironi, L., \& Narayan, R. 2014b, ApJ, 797, 47 
Herrmann, K. A., Hunter, D. A., \& Elmegreen, B. G. 2013, AJ, 146, 104

Holland, J. G., Böhringer, H., Chon, G., \& Pierini, D. 2015, MNRAS, 448, 2644

Hong, S. E., Ryu, D., Kang, H., \& Cen, R. 2014, ApJ, 785, 133

Hong, S. E., Kang, H., \& Ryu, D. 2015, arXiv:1504.03102

Inoue, S., Aharonian, F. A., \& Sugiyama, N. 2005, ApJL, 628, L9

Jee, M. J., White, R. L., Ford, H. C., et al. 2005, ApJ, 634, 813

Kang, H., \& Ryu, D. 2011, ApJ, 734, 18

Kang, H., Ryu, D., \& Jones, T. W. 2012, ApJ, 756, 97

Kang, H., \& Ryu, D. 2013, ApJ, 764, 95

Keshet, U., Waxman, E., Loeb, A., Springel, V., \& Hernquist, L. 2003, ApJ, 585, 128

Keshet, U., Waxman, E., \& Loeb, A. 2004a, JCAP, 4, 006

Keshet, U., Waxman, E., \& Loeb, A. 2004b, ApJ, 617, 281

Keshet, U., Kushnir, D., Loeb, A., \& Waxman, E. 2012, arXiv:1210.1574

Kitayama, T., Ito, Y., Okada, Y., et al. 2009, ApJ, 695, 1191

Kubo, J. M., Stebbins, A., Annis, J., et al. 2007, ApJ, 671, 1466

Kushnir, D., \& Waxman, E. 2009, JCAP, 8, 002

Kushnir, D., \& Waxman, E. 2010, JCAP, 2, 025

Laporte, C. F. P., White, S. D. M., Naab, T., \& Gao, L. 2013, MNRAS, 435, 901

Lau, E. T., Nagai, D., Avestruz, C. et al. 2015, ApJ, 806, 68

Loeb, A., \& Waxman, E. 2000, Nature, 405, 156

Matsukiyo, S., Ohira, Y., Yamazaki, R., \& Umeda, T. 2011, ApJ, 742, 47

McDonald, M., Bayliss, M., Benson, B. A., et al. 2012, Nature, 488, 349

Mantz, A. B., Abdulla, Z., Carlstrom, J. E., et al. 2014, ApJ, 794, 157

Menanteau, F., Hughes, J. P., Sifón, C., et al. 2012, ApJ, 748,7

Miniati, F. 2002, MNRAS, 337, 199

Miniati, F. 2003, MNRAS, 342, 1009

Miniati, F. 2015, ApJ, 800, 60

Montes, M., \& Trujillo, I. 2014, ApJ, 794, 137

Nelson, D., Genel, S., Pillepich, A., et al. 2015, arXiv:1503.02665

Nishino, S., Fukazawa, Y., Hayashi, K., Nakazawa, K., \& Tanaka, T. 2010, PASJ, 62, 9

Ota, N., Nagayoshi, K., Pratt, G. W., et al. 2014, A\&A, 562, A60

Parrish, I. J., McCourt, M., Quataert, E., \& Sharma, P. 2012, MNRAS, 419, L29

Patej, A., \& Loeb, A. 2015, ApJL, 798, L20

Petrosian, V. 2001, ApJ, 557, 560

Petrosian, V., Bykov, A., \& Rephaeli, Y. 2008, Sp. Sci. Rev., 134, 191

Presotto, V., Girardi, M., Nonino, M., et al. 2014, A\&A, 565, A126

Rephaeli, Y., Nevalainen, J., Ohashi, T., \& Bykov, A. M. 2008, Sp. Sci. Rev., 134, 71

Rudick, C. S., Mihos, J. C., \& McBride, C. K. 2011, ApJ, 732, 48

Ryu, D., Kang, H., Hallman, E., \& Jones, T. W. 2003, ApJ,
593, 599

Ryu, D., Kang, H., Cho, J., \& Das, S. 2008, Science, 320, 909

Sarazin, C. L. 1999, ApJ, 520, 529

Sari, R., Piran, T., \& Narayan, R. 1998, ApJL, 497, L17

Schaal, K., \& Springel, V. 2015, MNRAS, 446, 3992

Schlickeiser, R., Sievers, A., \& Thiemann, H. 1987, A\&A, 182, 21

Sheldon, E. S., Johnston, D. E., Masjedi, M., et al. 2009, ApJ, 703, 2232

Skillman, S. W., Xu, H., Hallman, E. J., et al. 2013, ApJ, 765,21

Stanford, S. A., Brodwin, M., Gonzalez, A. H., et al. 2012, ApJ, 753, 164

Takizawa, M., \& Naito, T. 2000, ApJ, 535, 586

Takizawa, M. 2002, PASJ, 54, 363

Takizawa, M. 2008, ApJ, 687, 951

Totani, T., \& Kitayama, T. 2000, ApJ, 545, 572

Tozzi, P., Santos, J. S., Jee, M. J., et al. 2015, ApJ, 799, 93

van Dokkum, P. G., Abraham, R., \& Merritt, A. 2014, ApJL, 782, L24

Vazza, F., Eckert, D., Brueggen, M., \& Huber, B. 2015, arXiv:1505.02782

Vink, J. \& Laming, J. M. 2003, ApJ, 584, 758

Watson, W. A., Iliev, I. T., D'Aloisio, A., et al. 2013, MNRAS, 433, 1230

Waxman, E., \& Loeb, A. 2000, ApJL, 545, L11

Williamson, R., Benson, B. A., High, F. W., et al. 2011, ApJ, 738, 139

White, S. D. M. 1994, arXiv:astro-ph/9410043

Yamada, K., \& Kitayama, T. 2005, PASJ, 57, 611

Zandanel, F., \& Ando, S. 2014, MNRAS, 440, 663 